\title{
Submission Type Code
}

National Cancer Institute

\section{Source}

National Cancer Institute. Submission Type Code. NCI Thesaurus. Code C94164.

A coded value specifying the kind of submission. 